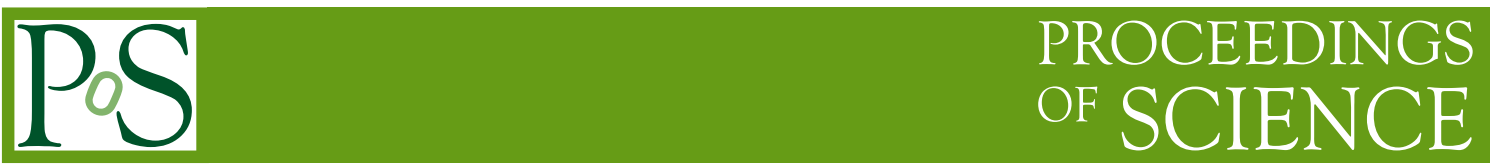

\title{
Angular Distribution of Solar Gamma Rays and Solar Neutrons Simulated by GEANT4 Program
}

\author{
K. Kamiya ${ }^{1)}$, K. Koga ${ }^{1)}$, S. Masuda ${ }^{2)}$, H. Matsumoto ${ }^{1)}$, Y. Muraki ${ }^{2)}$, S. \\ Shibata $^{3)}$, and Y. Tanaka ${ }^{4)}$
}

1) Tsukuba Space Centre, JAXA, Tsukuba 305-8505, Japan

2) Institute for Space-Environment Research, Nagoya University, Nagoya 464-8601, Japan

3) Engineering Science Laboratory, Chubu University, Kasugai 487-0027, Japan

4) Department of Physics, Hiroshima University, Hiroshima 739-8526, Japan

E-mail: kamiya.kohkiejaxa.jp

\begin{abstract}
When the accelerated protons hit the solar surface, high-energy gamma-rays and neutrons are produced. However observed intensity of gamma-rays and neutrons at the Earth depends on not only the position of the flare on the solar surface but also the direction of the accerelated protons and helium ions. Without precise knowledge of the angular distribution of secondary particles, we cannot estimate correct intensity of accelerated ions. Therefore we have made a Monte Carlo simulation based on the GEANT 4 program to understand the angular distribution induced by the proton-solar atmosphere and the helium-solar atomosphere collisions.

The collision processes of the accelerated ions with the solar atmosphere are simulated in the energy range of ions for $\mathrm{E}_{\mathrm{p} \text { or } \mathrm{He}}=0.1-100 \mathrm{GeV}$. The intensity of secondary neutral particles such as gamma-rays and neutrons are calculated as a function of the energy and emission angle $(\theta)$ to the incident protons or helium ions. The results of the Monte Carlo calculation are compared with actual simultanious observation of neutrons and gammarays by the two detectors, being detected by the SEDA-FIB neutron detector on board the ISS and by the LAT high-energy gamma-ray detector on board the FERMI satellite. The secondary neutrons with energy of $E_{n} \geq 35 \mathrm{MeV}$ and secondary gamma-rays with energy of $E_{\gamma} \geq 100 \mathrm{MeV}$ are investigated for comparison with actual data of observation.
\end{abstract}

Bexco, Busan, Korea

Presented by K. Kamiya 


\section{Introduction: the research purpose}

In the solar cycle 24, the the LAT detector (Large Area Telescope) onboard the FERMI satellite has observed several high-energy gamma-ray events. According to their report the number of events beyond $3 \sigma$ of the statistical signicance amounted to 26 . On the otherhand, the SEDA-FIB neutron detector onboard the International Space Station (ISS) obsreved 21 events of solar neutrons beyond the statistical sginificance of $3 \sigma$. Within them 11 events were detected by both detectors, however in another cases either neutrons or gamma-rays were detected by only one detector. In order to understand the reason for the difference, we have simulated the collision process of the accelerated protons and helium ions with solar atmosphere by the GEANT4 Monte Carlo program.

This paper consists of as follows. In Section 2 we describe the details of the Monte Cralo calculation. In Section 3 we present the angular distribution of the secondary particles as a function of the emission angle $(\theta)$ from the incident protons and the results of the incidence of the helium ions are provided in sub-section 3-1. In section 4 we compare the results with actual observations and summarize the results.

\section{Details of the Monte Cralo calculation by the GEANT4 program}

First we prepared the standard atmosphere in the simulation space that spreds $10^{7} \mathrm{~km}$ to the horizontal plane and protons were injected at the center of the plane from $2,000 \mathrm{~km}$ above the photosphere. One million protons were injected for each case. The energy and the emitted angle $(\theta)$ of ecah secondary with the energy higher than $30 \mathrm{MeV}$ was registered,( i.e., we record the neutral secondary particles with energy of $E_{n}$ and $E_{\gamma}$ $30 \mathrm{MeV})$. Then we have selected neutrons with energy higher than $35 \mathrm{MeV}$. Because the SEDA-FIB can detect neutrons with the energies higher than $35 \mathrm{MeV}$. On the other hand, for gamma-rays, taking account of the threshold energy of the FERMI-LAT, we have selected gamma-rays with energy higher than $100 \mathrm{MeV}\left(\mathrm{E}_{>}>100 \mathrm{MeV}\right)$.

The energy of protons has been changed from $\mathrm{E}_{\mathrm{p}}=100,300,500 \mathrm{MeV}, 1,5,10,50$ and $100 \mathrm{GeV}$ and the simulation was made. For the helium ions similar energy was selected but for the incident energy per one nucleon of the helium ions. When the accelerated ions enter into the solar atmosphere vertically (with small pitch angle), the secondary particles emitted toward the forward direction. They are absorbed in the thick solar atmosphere. On the other hand when the accellerated ions pass the solar atmosphere horizontally, the secondary particles emitted to the forward direction will escape from the solar atmosphere and they are expected to reach the Earth.

The latter case corresponds to the situation when the acelerated ions enter into the solar atomosphere vertically, but with large pitch angles. A pictorial presentaion of the situation may be shown later in Figure A. Let us remind that a lot of high-energy particles are emitted toward forward direction in comparison with backward direction in high energies. To underastand this effect clearly, we prepared a special thin target with the thickness of $10 \mathrm{gr} / \mathrm{cm}^{2}$ and investigated the angular distribution of secondaries. Here we present the results of the MC calculations. 


\section{The angular distribution of gamma-rays and solar neutrons}

Figure 1 presents the angular distribution $\mathrm{dN} / \mathrm{d} \Omega$ of the neutral secondary particles emitted by the protons with the incident energy of $500 \mathrm{MeV}, 1 \mathrm{GeV}, 5 \mathrm{GeV}$ and $10 \mathrm{GeV}$ respectively. The vertical axis is normalized at "per an incident proton".
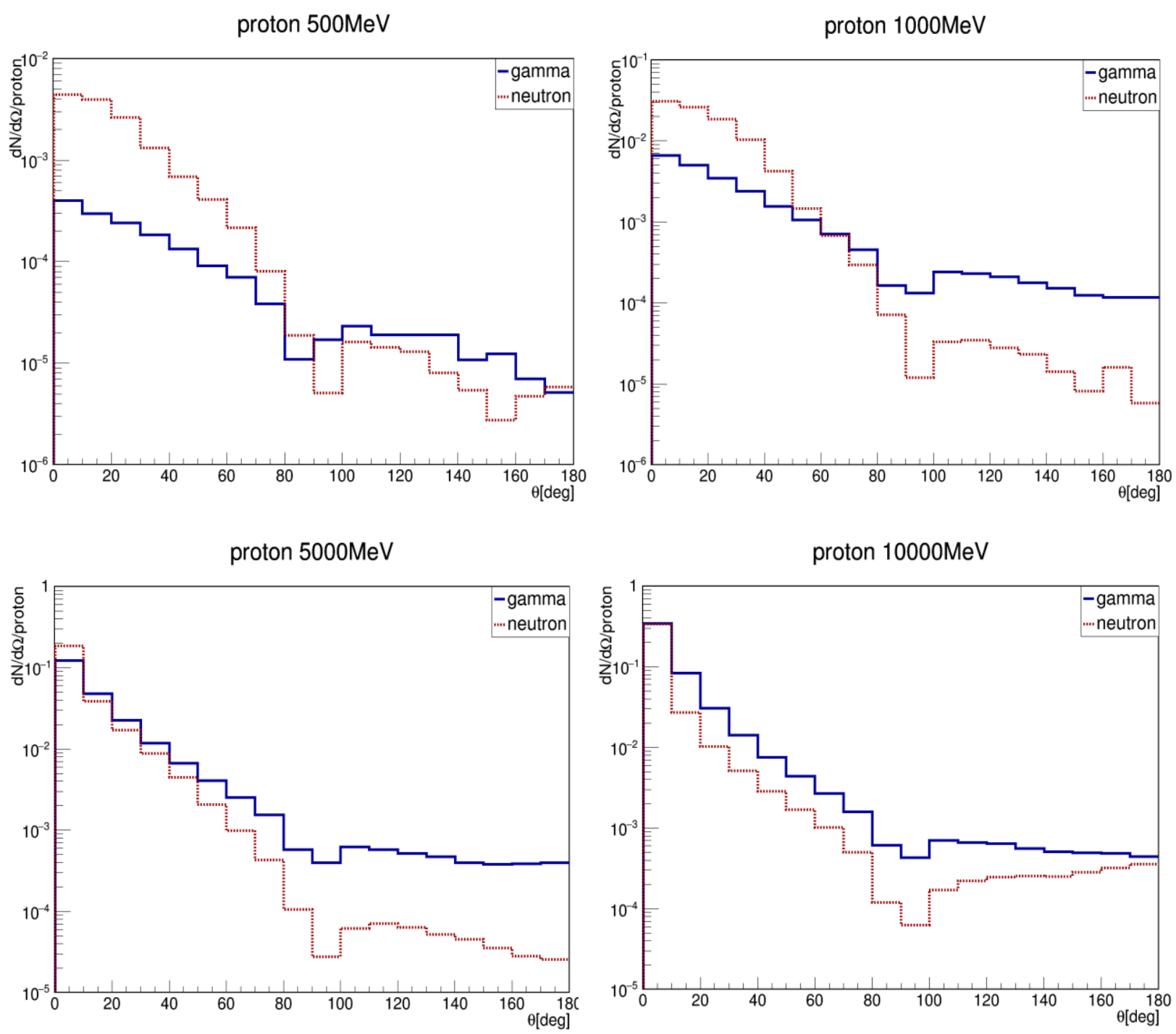

Figure 1. The angular distribution of neutrons (red dotted line) and gamma-rays (blue line). From top left for the protons with energy $\mathrm{Ep}=500 \mathrm{MeV}, 1 \mathrm{GeV}, 5 \mathrm{GeV}$ and 10 $\mathrm{GeV}$ respectively. When the incident energy increases the number of neutrons and gamma-rays also increases. This is due to increase of the secondary particles with collision energies. The vertical axis is presented by the unit of $\mathrm{dN} / \mathrm{d} \Omega$ [events/proton/acceptance]. According to the experimental results of the protonproton collisions at $\mathrm{E}_{\mathrm{p}}=20$ and $100 \mathrm{GeV}$, the average nutral pion multiplicities are reported as $<\mathrm{n}_{\pi 0}>\sim 2$ and 3 respectively. 
Figure 2 shows the intensity of the secondary particles for respective angle to the incident protons. The vertical axis is again normalized to "per one proton" and the number presents $(1 / 2 \pi \sin \theta)^{*} \mathrm{dN} / \mathrm{d} \theta$ or $\mathrm{dN} / \mathrm{d} \Omega$.

Figure 3 represents the flux of the secondary particles for respective angle to the incident helium ions. The vertical axis is again normalized to "per one nucleon" of the helioum nuclei and the number presents $\mathrm{dN} / \mathrm{d} \Omega$.

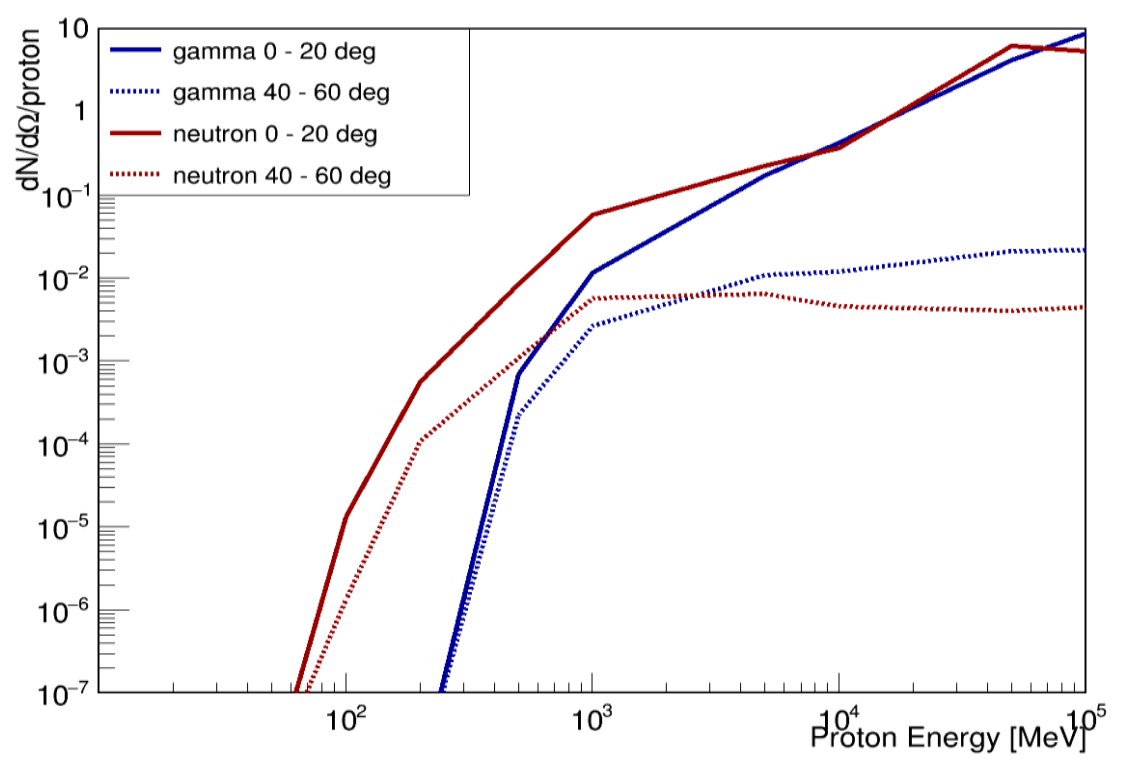

Figure 2-1. The flux of neutrons (red) and gamma-rays (blue) is presented as a function of the incident proton energy for the forward direction $\left(\theta=0-20^{\circ}, 20-40^{\circ}\right)$. In general the intensity of neutrons exceeds that of gamma-rays in the energy range less than a few $\mathrm{GeV}$ of the proton energy, $\mathrm{E}_{\mathrm{p}}$.

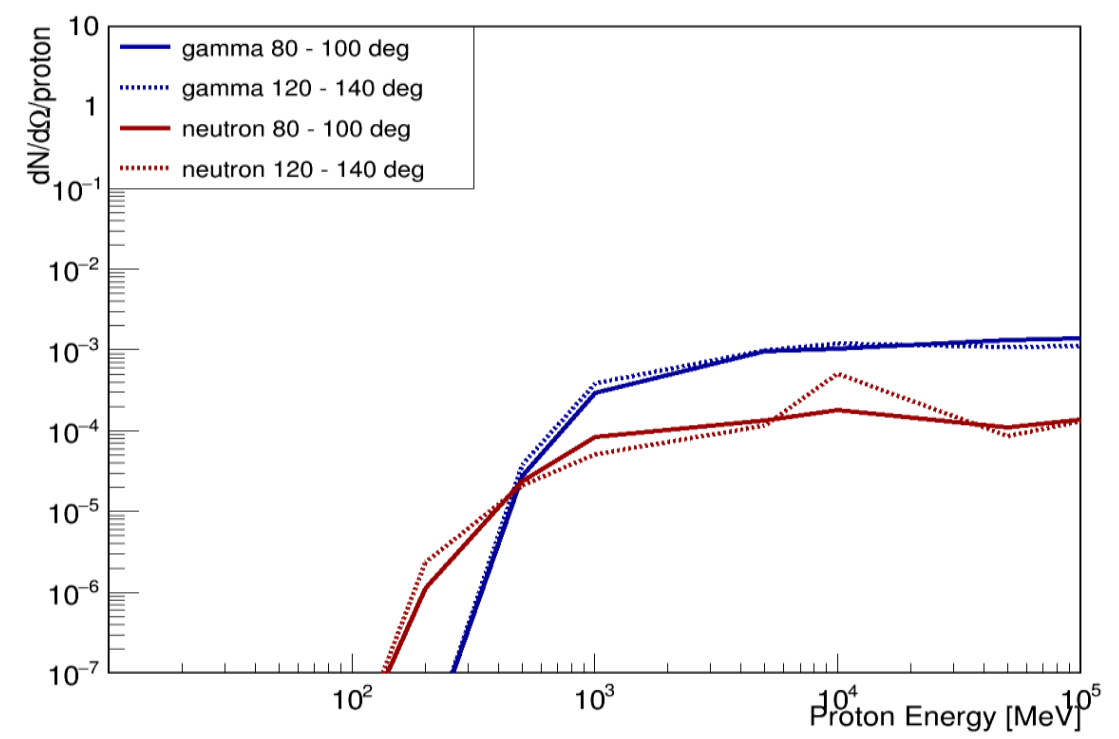

Figure 2-2. The flux of neutrons (red) and gamma-rays (blue) is presented as a function of incident proton energy for the backward direction $\left(\theta=80-100^{\circ}, 120-140^{\circ}\right)$. General speaking, the intensity of neutrons is lower than that of gamma-rays in the energy range higher than $\sim 400 \mathrm{MeV}$ of the proton energy. 
It is clear from Figures 1 and 2, that the ratio of $n / \gamma$ is different between the forward direction $\left(\theta=0^{\circ}-60^{\circ}\right)$ and the backward direction $\left(\theta=120^{\circ}-140^{\circ}\right)$. In the forward direction the $n / \gamma$ ratio exceeds beyond $\geq 10$, while in the backward direction the $n / \gamma$ ratio is suppressed to $\leq 0.1$. In this graph we have included secondary neutrons and gammarays with energy greater than $35 \mathrm{MeV}$ and $100 \mathrm{MeV}$ respectively, which correspond to the actual threshold of each detector.

\section{3-1. The angular distribution induced by Helium ions}

\section{$\mathrm{He} 500 \mathrm{MeV} / \mathrm{n}$}

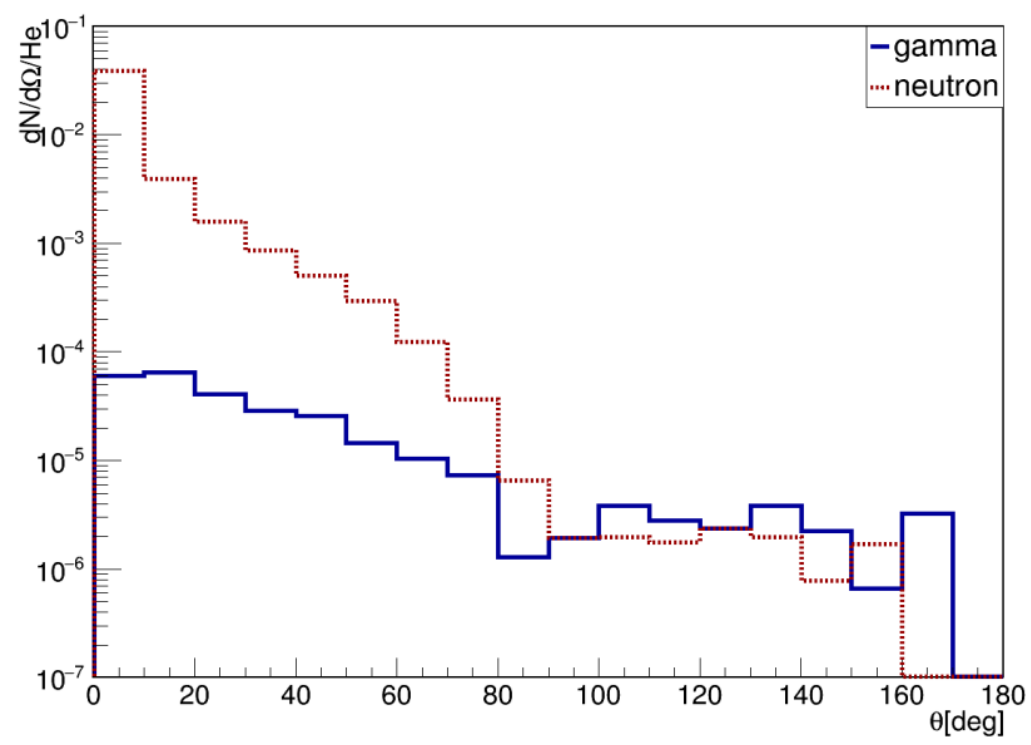

$\mathrm{He} 5000 \mathrm{MeV} / \mathrm{n}$

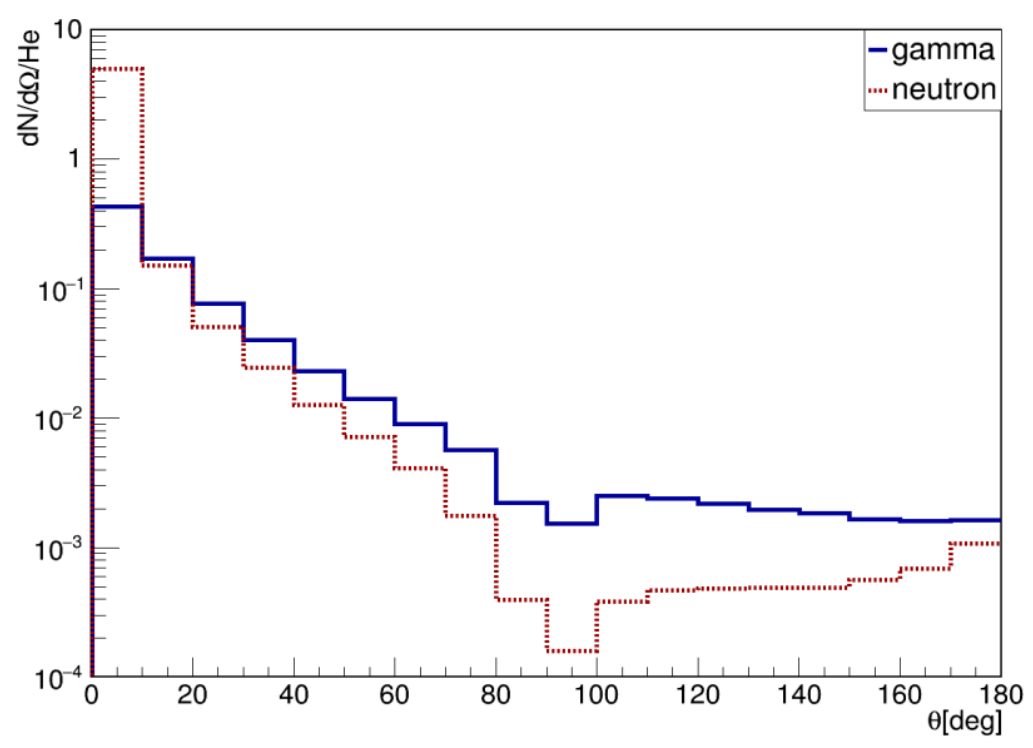

Figure 3-1. The angular distribution of gamma-rays and neutrons induced by the helium ions for the incident energy of $500 \mathrm{MeV}$ and $5 \mathrm{GeV} /$ nucelon. 
When the energy of helium ions increases, the difference of the distribution between the proton and helium incidences becomes less apparent. However in the energy range of the helium nuclei less than $500 \mathrm{MeV}$, the difference of the two angular distributions between gamma-rays and neutrons beomes remarkable. Since the energy threshold per nucleon approaches down to the threshold of pion production, the difference of the two distributions has become quite apparent. So the neutron rich events may be induced by the accelerated helium nuclei with rather soft energy spectrum. In order to demonstrate this tendency, in the next figures, we present two cases for different energies.

Figure 3-2 present the angular distribution of neutrons for the low energy incidence of protons and helium nuclei. For comparison of both graphs, the vertical scales of protons are adjusted as the same scale of the helium nuclei. For the helium incidence, at the zero degrees $\left(\theta=0-10^{\circ}\right)$, approximately $\sim 0.07$ neutrons are expected to be produced per nucleon. On the other hand in the proton incidence, within $\theta=0-10^{\circ}$, only $\sim 0.0003$ neutrons will be produced. The M.C. calculation tells us that at low energies, about $\sim 250$ times more abundant production of neutrons are expected to the helium neclei. The situation may be not so surprising. Because neutrons in the helium nucleus are accelerated together with protons with the same momentum and by the collisions with the solar atmosphere each nucelon is easily separated into four nucleons. Two of the four nucleons in the helium nucleus are neutrons. They are directly emitted into the forward direction.
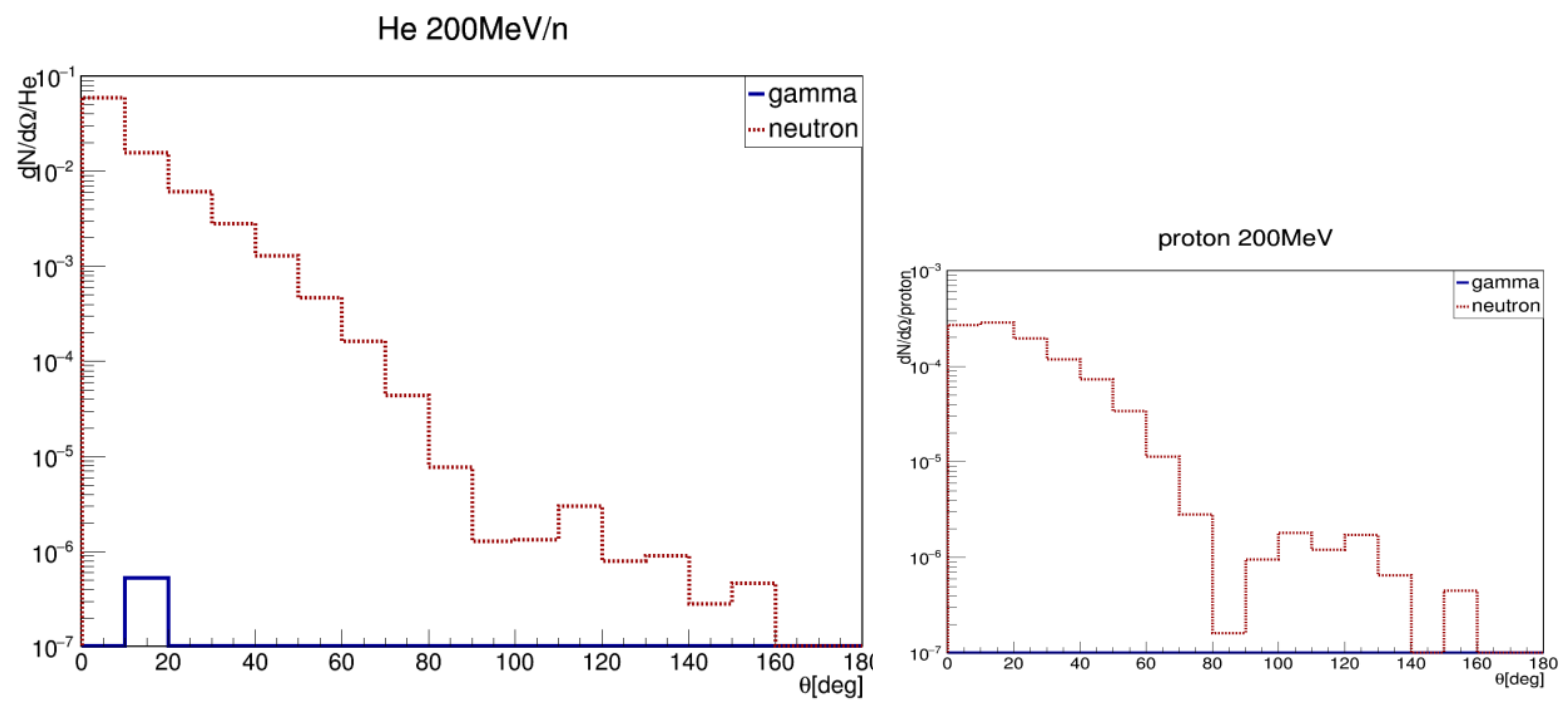

Figure 3-2. Comparison with the angular distribution of neutrons produced by the proton- and heium-solar atmosphere collisions for the incidenct energy of $\mathrm{E}_{\mathrm{p}}=200 \mathrm{MeV}$ or $\mathrm{E}_{\mathrm{He}}=$ $200 \mathrm{MeV} /$ nucleon. To compare with each flux, the vertical scale of the proton incidence is adjusted to the same scale of the helium incidence. Only one photon production is recorded for the helium incidence per one million helium nucleus entrance. 


\section{Discussions and Summary}

Let us compare the results with actual data. We examine present results with the two very impulsive flares observed on (1) 2010 June 10, (2) 2012 June 3 and another evnt observed on (3) 2011 March 7. The details of each event are published in this proceeding; for (1) and (2) in the paper of SH115 [1] and for (3) in the paper SH045 [2] respectively. The siganls of high-energy gamma-rays and neutrons were detected by the FERMI-LAT and SEDA-FIB detectors in cases of (1) and (2). The $n / \gamma$ ratios were $\sim 230$, $\sim 700$ and $\sim 1.7$ respectively.

For the very impulsive flares like (1) and (2), if the helium nuclei are dominantly accelerated beyond a few $\mathrm{GeV}$ togethr with protons and if they are bombard to the solar atmosphere almost horizontally, then we may have a solution to explain these high $n / \gamma$ ratios and the exsitence of high-energy gamma-rays with the energies of $\leq 400 \mathrm{MeV}$.

They will be described by the combination of the composition of the $\mathrm{H} / \mathrm{He}$ ratio and the enegy spectrum of the incident ions. Here we remind of a past report on the impusive flares and gardual flares summarized by Don Reams ${ }^{[3]}$. Accoding to him, the $\mathrm{H} / \mathrm{He}$ ratio is $\sim 10$ for the impulsive flare, while in the gradual flare the value is estimated as to be $\sim 100$. It would be a challenging subject to simulate the actual case, taking account of the composition of $\mathrm{H} / \mathrm{He}$ and changing the power index $(\gamma)$ of the accelerated ions $\left(\mathrm{E}_{\text {ions }}{ }^{-\gamma}\right)$ from $\gamma=2-6$. We shall present the results at the poster session of the conference and sumbit the final result at PoS.

About the case (3), the situation may be slightly different from (1) and (2). Becasue the observations of gamma-rays at the peak times were made not by the FERMI-LAT detector but by the ground based solar neutron telescope. The first impulsive flare was observed during 19:58-20:06 UT in 25-50keV and 19:41-20:10 UT in the X-ray region of $12-25 \mathrm{keV}$. The second peak shone for 5 minutes around 20:37UT. So the case (3) is not purely impulsive flare like cases (1) and (2) that conitnued only one minute. We could not confirm that the case (3) may be calssified into the gradual flare. Because we cannot see the shock front. However the SDO/AIA images strongly indicate that the bright point is located at quite high altitutde from the solar surface. We can explain the observed $n / \gamma$ ratio by a possible high energy proton bombardment onto the solar surface. This assumption is also consistent with present MC results presented in Figre 2-1.
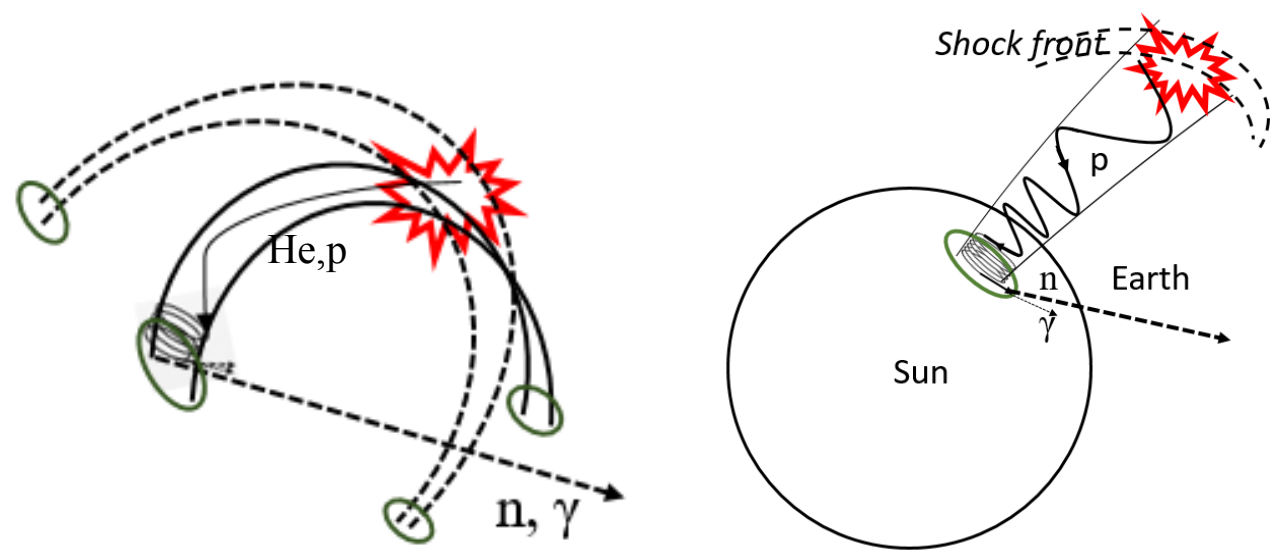

Figure A A schematic picture of the collisions between the accelerted ions and solar atomosphere. Left side view presents the Cases (1) and (2), while the right side picture reppresents the case (3). Active region is shown by the green circles. 
Additional information for the thickness of the target with $188 \mathrm{gr} / \mathrm{cm}^{2}$. proton $5000 \mathrm{MeV}$
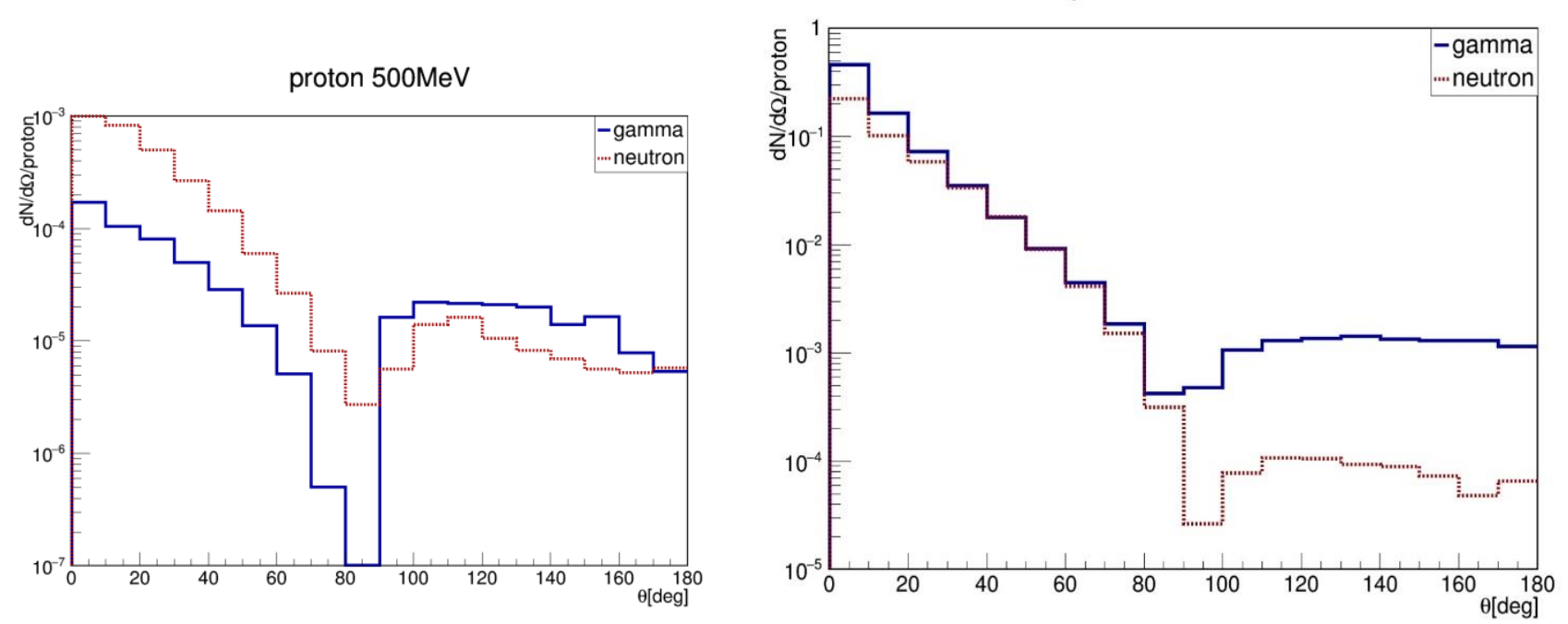

Figure B. These figures represent the anglular distribution of emitted gamma-rays and neutrons for the incident proton energy of $500 \mathrm{MeV}$ and $5 \mathrm{GeV}$. However the values are given for the thickness of the target of $188 \mathrm{gr} / \mathrm{cm}^{2}$. In comparison with that of $10 \mathrm{gr} / \mathrm{cm}^{2}$ as presented in Figure 1, we undertnad that the supression of neutrons at $\sim 0$ degrees is expected as to be $\wedge 40$ times less intensive, however the other parts of teh cross-section do not change remarkably.
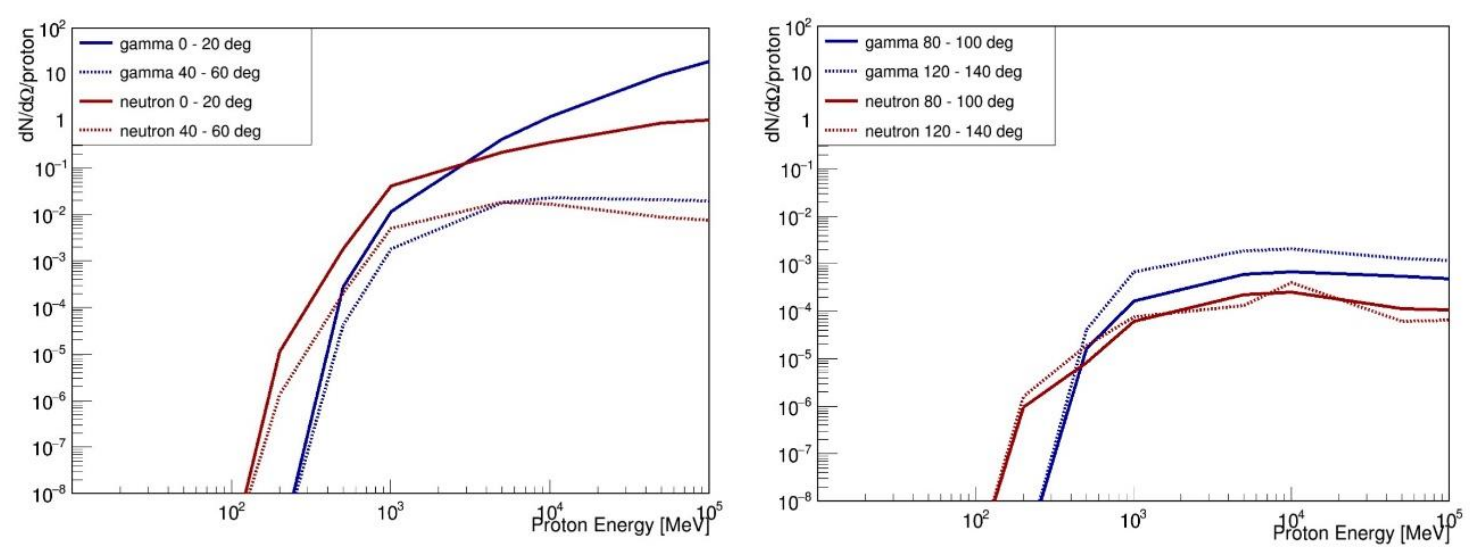

Figure C. Above figures present the angular distribution of neutrons ang gamma-rays. The left side figure represents for teh forward direction and the right side for the cbackward direction. In comparison with that of $10 \mathrm{gr} / \mathrm{cm}^{2}$ (Figure 2), except the supression in the low energy region of the forward direction, the genral tendency of the productionattenuation curve does not change so much .

\section{References}

[1] K.Kmaiya et al, This conference proceeding paper number SH115.

[2] Y. Muraki et al., This conference Proceeding paper number SH045.

[3] D. Reames, Review of Geophysics, Suppliment, 33 (1995) 585-589. 95RG00188. 